\title{
Questions de vie ou de mort
}

\section{Jean Martin}

Dr méd., membre de la rédaction

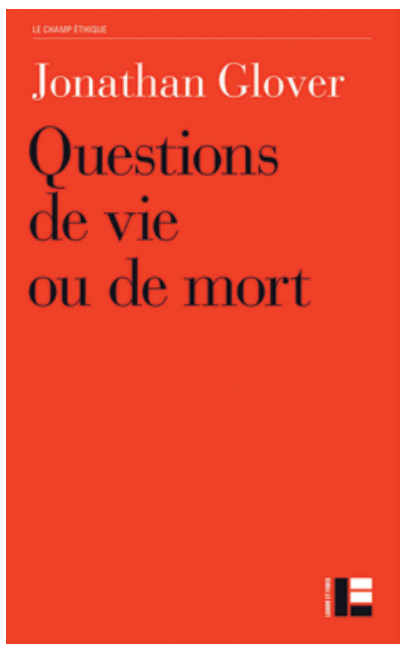

Jonathan Glover

Questions de vie ou de mort

Genève: Labor et Fides; 2017.

386 pages. $29 \mathrm{CHF}$.

ISBN 978-2-8309-1615-7

Pour qui s'intéresse à la bioéthique, les Editions Labor et Fides offrent une possibilité de se familiariser avec le travail majeur du philosophe britannique Jonathan Glover (1941). Ce qui surprend, c'est que la publication originale (Causing Death and Saving Lives) date de 1977, mais le propos n'a pour l'essentiel pas vieilli. Dans les débuts de mon intérêt pour les enjeux éthiques en biomédecine, j'avais été frappé par un autre titre de cet auteur, demandant quel genre de personnes nous devrions vouloir... [1].

Ce livre est une somme sur les questions «qui se posent lorsque l'on envisage d'éliminer ou, au contraire, de sauver des vies humaines» - première ligne de la préface, qui pourra faire froncer le sourcil (voire qui détournera de la lecture - ce serait une erreur). Tout en restant pluraliste, l'auteur présente des conceptions représentatives de la bioéthique anglo-saxonne d'orientation conséquentialiste/utilitariste - à laquelle sont réticents ceux en Europe continentale dont l'approche est très déontologique et qui tendent à appliquer les principes indépendamment de leurs effets. Quatrième de couverture: Glover montre «ce que peut être la phi- losophie morale appliquée lorsqu'elle tente de se frayer une voie hors des deux écueils que sont le relativisme et le dogmatisme».

Un large éventail de réflexion. «Nos attitudes à l'égard du suicide, de l'euthanasie, de la peine de mort et de la guerre ne peuvent pas être traitées rationnellement si on les considère de façon radicalement séparée les unes des autres.» Etonnant... fondamentalement correct. Le but est «d'aboutir à un système de réponses non contradictoires couvrant l'ensemble des questions relatives au faire mourir, en excluant les formes opposées d'absolutisme éthique» que sont l'interdiction totale et la permissivité totale. Ceci en rappelant que «faire mourir l'autre» est largement admis dans certaines circonstances (légitime défense, pour beaucoup en cas de guerre, et - de plus en plus refusée - peine de mort). Glover propose trois raisons fondamentales de condamner l'homicide en général: 1) il est immoral d'écourter une vie valant la peine d'être vécue; 2 ) il est immoral de faire mourir quiconque désire continuer à vivre; 3) toutes choses égales par ailleurs, il est moral de privilégier la décision ayant les meilleures conséquences pour le plus grand nombre.

A propos de 1) ci-dessus, l'auteur discute souvent dans les divers volets de son étude de la notion de «vie digne d'être vécue», qui suscite chez certains la réserve voire le rejet. Même si ce thème semble de la nature d'un indécidable, il doit à mon sens être débattu de manière différenciée, pondérée; d'autant plus aujourd'hui qu'il y a 40 ans, vu les défis voire les crises liées aux évolutions récentes de la médecine, de la maladie, du mourir.

Les thèmes principaux. Glover distingue deux grandes catégories: les problèmes émergeant dans le contexte médical (il y inclut l'attribution des ressources qui ne sont disponibles qu'en faible quantité) et ceux qui se posent dans des contextes socio-politiques généraux: guerre, peine de mort - discutant aussi la question de la grève de la faim, en adoptant l'attitude acceptée aujourd'hui que la personne capable de discernement ne doit pas être alimentée de force (voir p. 201-209).

Trois parties à l'ouvrage. La première traite des méthodes et des limites de l'argumentation morale. La deuxième des théories morales, dont: la doctrine de la vie sacrée (à laquelle l'auteur n'adhère pas - il lui pré- 
fère une approche fondée sur le respect de l'autonomie des personnes et sur la qualité de la vie qu'elles mènent); les individus réels et les individus potentiels; la question des fins et des moyens (théorie du double effet); celle de l'inaction et de l'indifférence (actes et/vs omissions).

La troisième partie (deux tiers du livre), «Problèmes d'éthique appliquée», aborde d'abord les sujets classiques: l'avortement - du point de vue du fotus (quand devient-on une personne?) et du point de vue des femmes et de leurs droits*; l'infanticide; le suicide; l'euthanasie (avec ou sans demande/consentement du patient concerné); la sélection des personnes/patients. Depuis la parution princeps de l'ouvrage (note de J.M.), les transplantations d'organes et la procréation médicalement assistée notamment se sont ajoutées à cette liste, et les questions autour de la fin de vie se sont aiguisées, au vu des avancées permettant de maintenir longtemps une existence de type végétatif. Puis viennent des chapitres détaillés sur la peine de mort et sur la guerre.

Dans son travail, l'auteur apporte des références non seulement philosophiques mais aussi historiques et littéraires, tout en suivant de près les situations pratiques.

A propos de critères de nature conséquentialiste. Chapitre attendu dans une telle optique: «Le nombre de vies sauvées importe-t-il?» Autre considération, qui n'est attirante pour personne mais se pose aussi bien en clinique que pour les politiques publiques: «Il ne fait aucun doute que nous n'avons pas encore suffisamment réfléchi à la question de savoir combien notre société devrait être prête à dépenser en vue de sauver des vies.» On sait que la considération de critères sociaux, en particulier s'agissant de transplantation d'organes, est un sujet difficile. Les textes légaux exigent que la sélection d'un receveur ne soit faite que sur la base de critères médicaux. Toutefois, appliqué de manière étroite, cela mène à des situations mal acceptables du point de vue du simple bon sens (privilégier un malade de plus de 80 ans dont le dossier médical est marginalement plus «approprié» que celui d'une mère de famille de 35 ans...). Ce qui demande, et c'est justifié dans une optique de santé publique, que des paramètres du registre social soient inclus dans l'appréciation dite médicale. Glover: «Si la vie de deux personnes est en jeu, il convient de considérer comme un critère très important le nombre de personnes dont chacune à la charge [...] très bonne raison de ne pas laisser le hasard décider.» Plus avant: «Une fois admis d'accorder une importance aux personnes à charge, faut-il prendre en considération des effets plus généraux tels que la contribution de chacun envers la société? Il existe de bonnes raisons de ne pas choisir ce critère.» C'est bien la règle aujourd'hui, entre autres parce qu'on ne pourrait définir de critères consensuels sur la "valeur sociale» de tel ou tel.

Soins palliatifs et assistance au suicide. "Il n'est pas évident qu'il faille penser l'euthanasie [en Suisse, on parlerait ici d'assistance au suicide] en termes d'alternative aux soins palliatifs: pourquoi ne pas l'envisager comme un complément? Un hôpital dans lequel on pratique des euthanasies volontaires peut très bien avoir du personnel faisant tout ce qu'il peut pour rendre inutiles les demandes d'euthanasie.» Ce que Véronique Fournier appelle en 2016 soins palliatifs intégraux [2].

Point d'importance: «La question de savoir si l'on doit mettre en place un système très formel de validation des demandes d'euthanasie [ou suicide assisté]. D’un côté certains prétendent que des signatures de témoins fournissent des garanties. D'un autre, les procédures bureaucratiques constituent une interférence dans la relation unissant les patients aux médecins. Il me semble préférable d'avoir confiance dans l'idée que les médecins n'ôteront pas la vie de patients de façon injustifiée et [suivront des normes] de déontologie.» Il me paraît aussi (J.M.) que des procédures trop formalisées présenteraient des inconvénients sérieux (y compris en donnant l'impression d'une caution de l'Etat si une commission officielle avait le mandat d'examiner chaque cas - et ce serait là une vraie atteinte au caractère privé, personnel, de la démarche).

Prévention et santé publique. Au sujet de politiques de santé que Glover appelle interventionnistes: «Dans quelle mesure pouvons-nous légitimement recourir à la pression sociale ou à une législation paternaliste afin d'empêcher que des personnes risquent leur vie? Par exemple s'agissant du port de la ceinture de sécurité, de l'usage de drogues et du tabac, de l'obésité, des sports dangereux.» Débat bien connu. Ici, souligner la différence à faire selon la gravité potentielle de la limite mise à la réalisation de soi-même: ainsi des interdictions visant l'alpinisme seraient une atteinte bien plus sérieuse que le port de la ceinture de sécurité. Enfin, très discuté aujourd'hui, un sujet qui était moins urgent il y a 40 ans: celui des droits et du bien-être de ceux qui nous suivent, "de l'importance que nous devons accorder aux intérêts et à la liberté d'action des générations à venir».

\section{Références}

1 Glover J. What sort of people should there be? Harmondsworth: Penguin Books; 1984

2 Voir Martin J. Le long cheminement en France de la réflexion sur la fin de vie (à propos de "La mort est-elle un droit?», de Véronique Fournier ). Bull Méd Suisses. 2017;98(9):293.

\footnotetext{
cite «un décret du

condamnant tout

l'utérus, par exemple dan

alors que ne pas le faire

aboutissait à la mort de la
}

jean.martin[at]saez.ch 